\title{
Multiple recurrent arachnoid cysts with adhesive arachnoiditis following a spontaneous spinal subdural hematoma in an elderly patient: a case report with a literature review
}

\author{
Junhyeok Yang, Dal-Sung Ryu, Hong Yoon, Seung-Hwan Yoon \\ Department of Neurosurgery, Inha University College of Medicine, Incheon, Korea
}

\begin{abstract}
Although arachnoid cysts associated with adhesive arachnoiditis are extremely rare, they can have devastating clinical outcomes. This severe form of arachnoiditis develops from fibrosis of the arachnoid membrane with subsequent adhesion of the arachnoid space. An arachnoid cyst with adhesive arachnoiditis can compress the spinal cord. Surgical decompression can be considered in case of neurologic deficits. However, surgery should be chosen carefully because it does not always guarantee favorable clinical outcomes. We present a case of recurrent arachnoid cysts with adhesive arachnoiditis following a spontaneous spinal hematoma in an elderly woman who suffered from progressive myelopathy despite 2 adhesiolysis treatments.
\end{abstract}

Keywords: Arachnoiditis; Arachnoid cysts; Recurrence; Spinal subdural hematoma; Aged

\section{Introduction}

Adhesive arachnoiditis in addition to spinal arachnoid cysts is extremely uncommon [1]. It is known to be associated with devastating clinical outcomes. Although the exact pathophysiology associated with an arachnoid cyst remains unclear, congenital, inflammatory, iatrogenic, and post-traumatic factors might play roles in its pathophysiology [2]. Severe diffuse inflammatory reaction along the whole spinal cord can occur rarely after hemorrhage [3]. Adhesion forms tethering of neural component including spinal cord and nerve roots. Subsequently, cerebrospinal fluid (CSF) location due to the disruption of flow is generated. It can compress the spinal cord with a clinical manifestation as progressive motor weakness. Previous reported cases were adhesive arachnoiditis following aneurysmal or spontaneous subarachnoid hemorrhage. In this article, we report a case of a recurrent spinal arachnoid cyst

Received: July 28, 2021

Revised: September 29, 2021

Accepted: October 1, 2021

Corresponding Author: Dal-Sung Ryu, MD, PhD

Department of Neurosurgery, Inha University Hospital, 27 Inhang-ro, Junggu, Incheon 22332, Korea.

Tel: +82-32-890-3596; Fax: +82-32-890-2374;

E-mail: dalsung.ryu@gmail.com with arachnoiditis following a spontaneous spinal subdural hematoma (SSDH) with a literature review.

\section{Case Report}

A 73-year-old female visited the emergency room with back pain and bilateral leg weakness a week ago. She did not have complains of any relevant pain. The patient denied any recent pain procedures including acupuncture and lumbar puncture. She was taking oral anti-hypertensive drugs. However, she did not receive any anticoagulant therapy.

Physical examination showed flaccid motor weakness (manual muscle test $2 / 2$ ) for both legs, decreased sensory perception below T11 dermatome bilaterally, both knee jerk hyperreflexia, and decreased rectal tone. The patient had saddle anesthesia and bladder retention. Laboratory tests including tests related to coagulation at the time of admission showed normal results: a platelet count of $246,000 / \mu \mathrm{L}$, a prothrombin time of 14.4 seconds (normal range, $11.0-15.0$ seconds), a partial thromboplastin time of 31.3 seconds (normal range, 29.0-44.0 seconds), and an international normalized ratio of 1.11 (range, $0.85-1.30$ ).

Plain radiographs and computed tomography (CT) scans revealed unremarkable mild spondylosis without a traumatic change 
of bony structures. Magnetic resonance image (MRI) of the whole spine showed long segmental subdural hematoma involving thoracic and lumbar spine lumbar spine (Fig. 1). Compression of spinal cord was severe at T9 level and moderate at T6-T7 levels. Subtle cord signal change was found at T9 and T10 levels. Prominent hematoma at left subdural space of T12 and L1 levels deviated spinal cord to the right side. Gadolinium enhance image did not reveal a tumor or vascular disease. Because some spinal subdural or subarachnoid hematomas are associated with cerebral lesions like subarachnoid hemorrhage due to the rupture of an aneurysm, CT and MRI of the brain were performed. However, their results were unremarkable without any evidence of acute hemorrhagic or infarct lesion. Results of cerebral angiography were un-
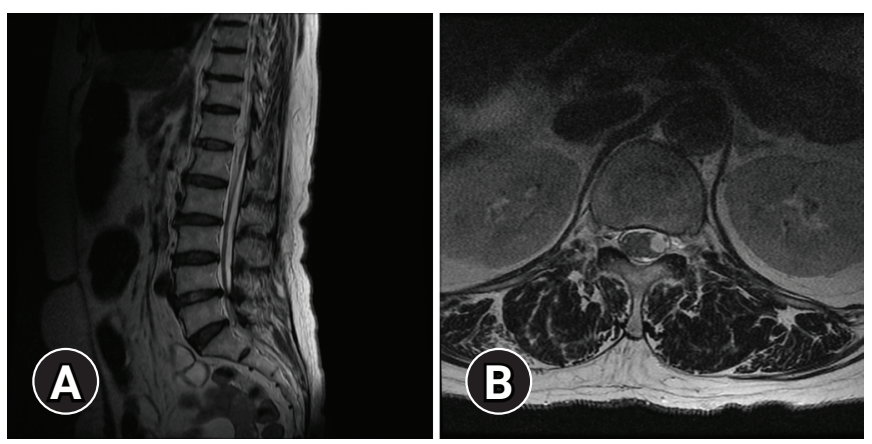

Fig. 1. Sagittal (T2-weighted $[A]$ ) and axial T2-weighted $[B]$ at the T12 level) magnetic resonance images revealing subdural hematoma extending from T9 to L1 and deviation of the spinal cord at T12 caused by the hematoma. Written informed consent was obtained for publication of this case report and accompanying images. remarkable as well.

Although surgical decompression was considered at the time of admission, the patient showed neurologic recovery in a short time. Thus, the patient was observed closely with intravenous injection of tranexamic acid 2,500 $\mathrm{mg}$ and vitamin $\mathrm{K} 10 \mathrm{mg}$ every 6 hours. Manual motor grade became greater than 4 for both legs at one day after admission. Fortunately, she was discharged from the hospital after the period of rehabilitation. At the time of discharge, the patient could walk without assistance.

However, 1 month later after the discharge, she visited the outpatient clinic with a recent gradual mild weakness of both legs and persistent urinary incontinence. MRI of the whole spine was taken immediately to evaluate the reason for neurologic deterioration. The exam showed multifocal arachnoid cyst with intradural adhesion, adhesive arachnoiditis at thoracic and lumbar spine resulting in multiple indentations and deviation of the spinal cord (Fig. 2AC). Multifocal intradural cystic lesions and compressive myelopathy were observed within the spinal cord at T6-T12 levels.

Surgical decompression was performed under general anesthesia. Lamina of the T3 segment, which showed the most severe compression of the spinal cord, was exposed with midline incision and subsequent periosteal dissection. Laminectomy was performed to visualize the thecal sac. Dura was opened under a microscope and retracted with a stay suture. There was a solitary and large cystic lesion covered by a very thin membrane (Fig. 3). The lesion filled with clear fluid was deviating the spinal cord into the left side with severe compression. Since en bloc resection was impossible, obtaining the internal fluid was decided for the diagnosis. About $5 \mathrm{~mL}$ amount of the internal fluid was aspirated with a $1 \mathrm{~mL}$
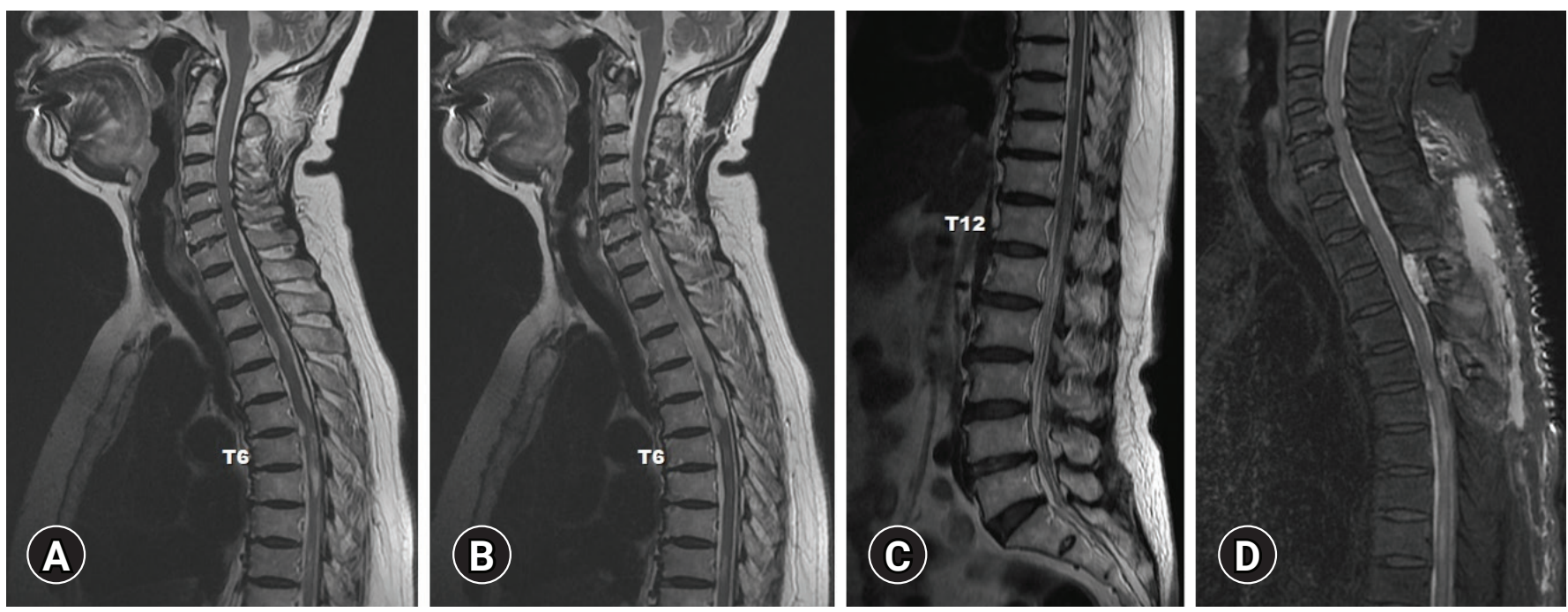

Fig. 2. (A-C) Follow-up sagittal (T2-weighted) magnetic resonance image (MRI) revealing a newly developed arachnoid cyst with arachnoiditis compared to previous MRI in Fig. 1. (D) Postoperative sagittal T2-weighted MRI revealing complete removal of cystic compression. Written informed consent was obtained for publication of this case report and accompanying images. 
syringe and subjected to laboratory tests. After the aspiration, the cyst was collapsed. The membrane could not be acquired because the texture was similar to the membrane as in cerebral chronic subdural hematoma. Because it was not certain that the cyst was connected to caudal segments, another laminectomy was conducted at the T5 level. With the dura opening, a similar membrane was noted. However, it was empty probably due to the prior aspiration. The spinal cord seemed to be unremarkable at both levels. Dura was closed with a continuous suture. Laminectomized bones were attached into the removed site with plates.

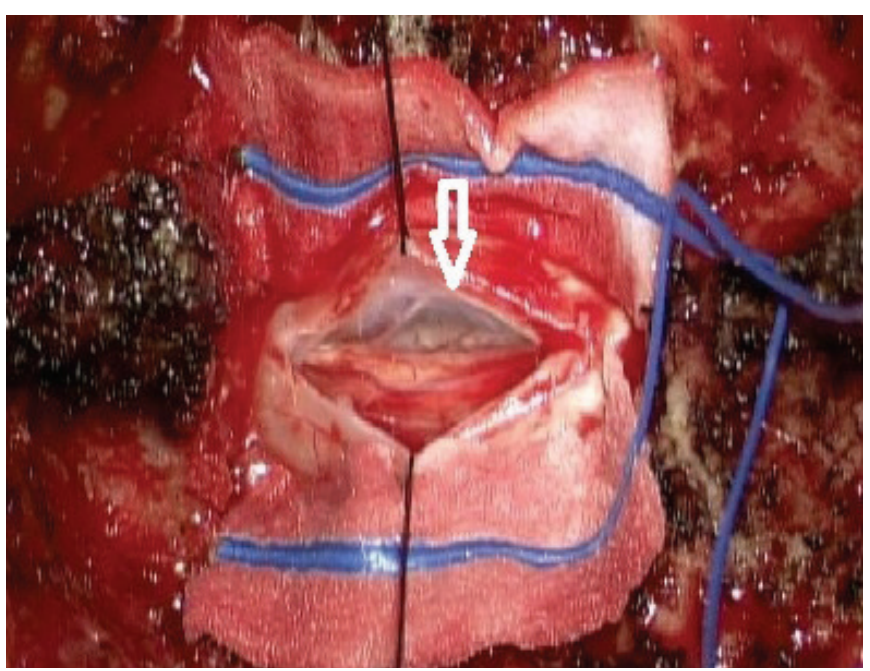

Fig. 3. A picture of the operative field after total laminectomy at T3, showing that the dura was opened and revealing a gray and cystic lesion (white arrow), from which clear fluid was aspirated with a syringe. Written informed consent was obtained for publication of this case report and accompanying images.
Microscopic exam of the aspirated fluid revealed CSF mixed with hemorrhage (red blood cell, 1,250/ $\mathrm{mm}^{3}$ ). Laboratory examination of the fluid showed glucose level of $50 \mathrm{mg} / \mathrm{dL}$, protein level of $23.0 \mathrm{mg} / \mathrm{dL}$, and lactic acid level of $1.68 \mathrm{mmol} / \mathrm{L}$. On both Gram-stain and bacterial culture, no microorganism was isolated. Parasite Ab-IgG to rule out neurocysticercosis was negative. Additional blood tests such as anti-streptolysin $\mathrm{O}$ test were negative.

The patient's motor function of both legs improved after the decompression. Postoperative MRI showed well removal of multiple arachnoid cysts along the thoracic spine seen in previous MRI (Fig. 2D). She was discharged at 1 week after surgery without any complication. At the first visit to the outpatient clinic 1 month later, her gait disturbance disappeared completely. However, she complained of progressive weakness of both legs again 5 months later. Thoracic spine MRI showed recurrent multiple arachnoid cysts with adhesive arachnoiditis at T3-T7 levels resulting in multiple indentations and left deviation of the spinal cord. Compressive myelopathy was also observed at T2 and T4 to T7 levels (Fig. 4A, 4B). At this time, wide decompression and adhesiolysis were tried to prevent the recurrence. Laminectomies were performed between T2 and T7 levels. Severe adhesion was observed at the moment of dura opening. There were multiple cystic lesions. Adhesiolysis and cystic wall removal was performed as much as possible. However, full decompression could not be accomplished due to decreased motor evoked potential (MEP) amplitude.

At postoperative MRI, a cystic lesion was not seen completely (Fig. 4C). However, her lower limb motor grade did not recover fully. She was discharged for rehabilitation with follow up in the outpatient clinic. Four months later, she complained of neurologic deterioration again. MRI scans revealed arachnoid cysts with se-
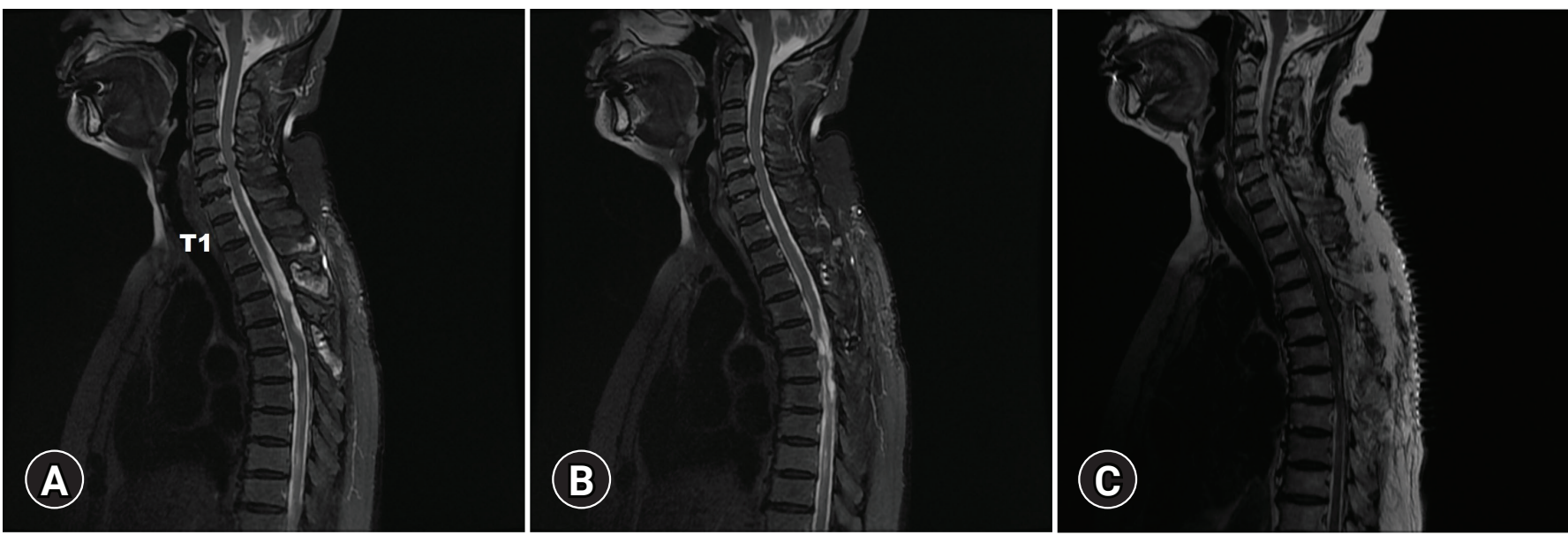

Fig. 4. (A, B) Follow-up sagittal (T2-weighted) magnetic resonance image (MRI) revealing a newly developed arachnoid cyst with arachnoiditis compared to previous MRI in Fig. 2. (C) Postoperative sagittal T2-weighted MRI revealing complete removal of cystic compression. Written informed consent was obtained for publication of this case report and accompanying images. 
vere spinal cord compression at more proximal level than previous laminectomy site (Fig. 5). A Surgical procedure with adhesiolysis and cyst-subarachnoid shunt was considered. However, close observation was decided instead of decompression after an in-depth discussion with the patient and her family members because we could not be convinced how to prevent a recurrence. Fortunately, the patient's motor function has not been worse for 6 months since then. She is currently wheelchair-bound with rehabilitation and regular follow-up.

\section{Discussion}

In this case, an intractable arachnoid cyst with arachnoiditis after a resolution of spontaneous spinal hematoma was demonstrated. Despite 2 times of surgical decompression, the debilitating clinical course failed to be overcome.

First, the subdural hemorrhage seen at the first spine MRI occurred spontaneously. Trauma is a common cause of spinal subdural hemorrhage. It is often associated with hemostatic disorders following an iatrogenic injury such as a spinal puncture or an anticoagulation therapy [2]. In this case, the patient denied any traumatic events and hemostatic problems such as polycythemia, leukemia, and hemophilia. She did not take any anticoagulants either.

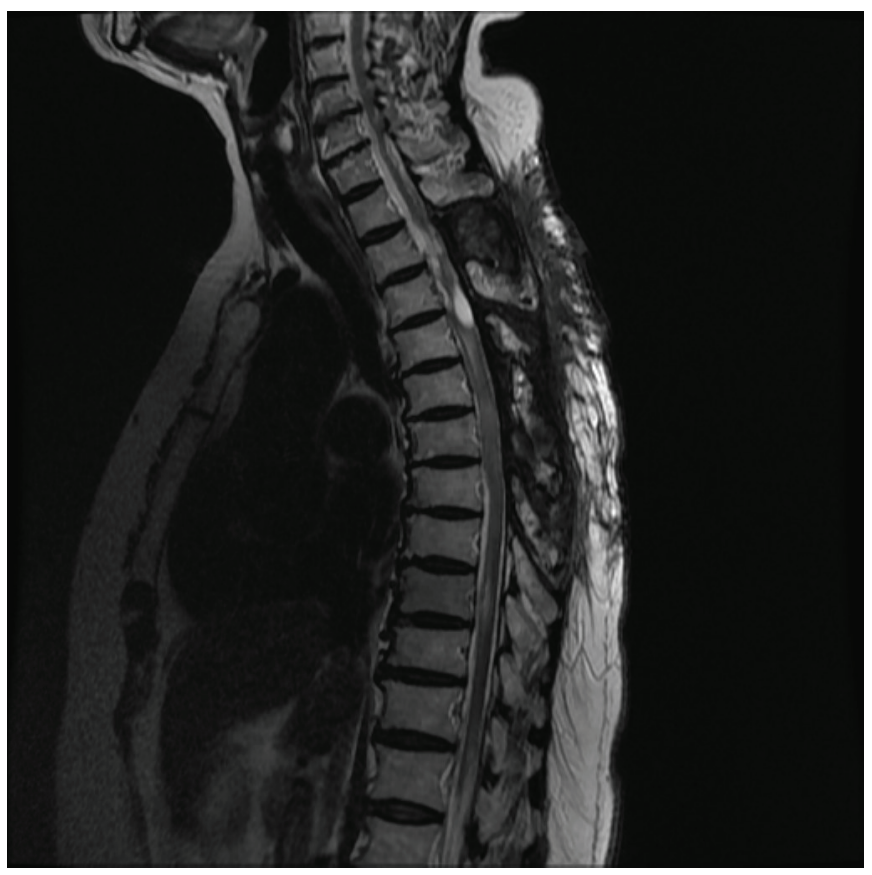

Fig. 5. Follow-up magnetic resonance image (MRI) at postoperative 4 months revealing recurrent arachnoid cyst with arachnoiditis compared to postoperative sagittal T2-weighted MRI. Written informed consent was obtained for publication of this case report and accompanying images.
Most spinal hematoma occurs in the epidural space or the subarachnoid space because spinal subdural space is narrow. And there is few angiogenesis and only has radiculomedullary vessels whereas a subarachnoid space contains major blood vessels and bridging veins [1]. A possible mechanism of $\mathrm{SSDH}$ is that the pressure of vessels increases due to rising thoracic or abdominal pressure in the subarachnoid space. As a result, blood vessels might have ruptured. Hemorrhage can be diluted by CSF and migrate into the subdural space when the arachnoid membrane ruptures [4].

Blood clots seem to cause fibrosis of the arachnoid with an inflammatory reaction. This increases collagen synthesis and accumulation of procollagen propeptides which lead to fibrosis in subarachnoid space [5]. Adhesive arachnoiditis can be associated with infection, trauma, and hemorrhage such as subarachnoid hemorrhage due to aneurysmal rupture [5]. Iatrogenic origin is also assumed after various procedures including spinal surgery, spinal tap, pain block, and spinal anesthesia [6,7]. Prolonged bed rest, use of fibrin glue, collapse of subarachnoid space due to lumbar puncture were also pointed out as risk factors of arachnoiditis [8]. Proposed mechanisms include persistent inflammatory reaction, occult meningitis, and collapse of the subarachnoid space causing pial adhesion. Arachnoid cyst might form when adhesion of arachnoid membrane acts as a one-way valve with CSF flow diversion [3]. Another hypothesis is that the cells of cyst wall is associated with the production of CSF. Continuous production of CSF after location is likely to form arachnoid cyst filled with CSF [9].

The exact diagnosis was unclear until the dura mater was opened at the first surgery. Liquefied hematoma with a migrated location was a strong differential diagnosis at that time. However, the fluid inside the membrane was CSF itself, not showing xanthochromatous color as in liquefied hematoma. Spinal cysticercosis was another differential diagnosis that often resembles other cystic diseases mostly located in the thoracic level. In our case, enzyme-linked immunosorbent assay was negative without an elevated protein. In addition, MRI did not show well defined T2 weighted image. Therefore, we could exclude spinal cysticercosis.

Medical treatment for arachnoiditis did not show improvement until now. Hackert et al. [10] reported improvement in one fourth patient after immunotherapies including corticosteroids, methotrexate, or plasmapheresis but firm clues seem to insufficient. There are several surgical options to treat an arachnoid cyst with adhesive arachnoiditis. Shikata et al. [11] have reported $54.8 \%$ to $80 \%$ of good results after nonmicroscopic and microscopic lysis. However, simple cyst fenestration was unhelpful to prevent the recurrence in this patient. During the revision surgery, wide decom- 
pression and adhesiolysis was tried. But it was not easy due to decreased MEP amplitude. Considering that the adhesion was much worse in the second operation, complete cyst wall removal and restoration of normal CSF circulation should have been performed rather than simple decompression at first. Although not performed in this case, myelogram CT may help to find communication between cyst and normal CSF when the result of MRI is insufficient [12]. Several authors even advocated conservative treatment because of devastating clinical results despite surgical decompression as in this case $[1,13]$. A successful result has been reported with CSF diversion with shunt by several authors [14]. A subarachnoid-subarachnoid shunt might be relatively simple way to create a bypass for CSF circulation than other passages although this procedure requires more clinical experience. Treatment should be planned depending on the patient's age, the presence of neurologic deficit, the location of the cyst, and the amount of spinal cord compression. More importantly, geriatric people are much frail to neurologic injury and surgical procedure. Obtaining informed consent after sufficient communication with the patient is the most important because relapse is common after surgical decompression in arachnoid cyst with adhesive arachnoiditis.

\section{Conclusion}

A 73-year-old female demonstrated repetitive relapse of arachnoid cysts with adhesive arachnoiditis following a spontaneous spinal hematoma. Surgical treatment including fenestration, cyst wall removal, adhesiolysis, or bypass can be tried after obtaining the patient's understanding sufficiently because a devastating clinical result is common due to recurrence.

\section{Conflicts of interest}

No potential conflict of interest relevant to this article was reported.

\section{ORCID}

Junhyeok Yang, https://orcid.org/0000-0002-5124-9872

Dal-Sung Ryu, https://orcid.org/0000-0003-0895-3431

Hong Yoon, https://orcid.org/0000-0001-5250-9747

Seung-Hwan Yoon, https://orcid.org/0000-0003-0558-2313

\section{REFERENCES}

1. Kok AJ, Verhagen WI, Bartels RH, van Dijk R, Prick MJ. Spinal arachnoiditis following subarachnoid haemorrhage: report of two cases and review of the literature. Acta Neurochir (Wien) 2000;142:795-8.

2. Yanni DS, Mammis A, Thaker NG, Goldstein IM. Traumatic fracture of thin pedicles secondary to extradural meningeal cyst. J Surg Tech Case Rep 2011;3:40-3.

3. Rahmathulla G, Kamian K. Compressive cervicothoracic adhesive arachnoiditis following aneurysmal subarachnoid hemorrhage: a case report and literature review. J Neurol Surg Rep 2014;75:e56-61.

4. Kyriakides AE, Lalam RK, El Masry WS. Acute spontaneous spinal subdural hematoma presenting as paraplegia: a rare case. Spine (Phila Pa 1976) 2007;32:E619-22.

5. Basaran R, Kaksi M, Efendioglu M, Onoz M, Balkuv E, Kaner T. Spinal arachnoid cyst associated with arachnoiditis following subarachnoid haemorrhage in adult patients: a case report and literature review. Br J Neurosurg 2015;29:285-9.

6. Na EH, Han SJ, Kim MH. Delayed occurrence of spinal arachnoiditis following a caudal block. J Spinal Cord Med 2011;34: 616-9.

7. Hirai T, Kato T, Kawabata S, et al. Adhesive arachnoiditis with extensive syringomyelia and giant arachnoid cyst after spinal and epidural anesthesia: a case report. Spine (Phila Pa 1976) 2012; 37:E195-8.

8. Taguchi Y, Suzuki R, Okada M, Sekino H. Spinal arachnoid cyst developing after surgical treatment of a ruptured vertebral artery aneurysm: a possible complication of topical use of fibrin glue. Case report. J Neurosurg 1996;84:526-9.

9. Basaldella L, Orvieto E, Dei Tos AP, Della Barbera M, Valente M, Longatti P. Causes of arachnoid cyst development and expansion. Neurosurg Focus 2007;22:E4.

10. Hackert J, Maßmann L, Sure U, et al. Immunotherapies in chronic adhesive arachnoiditis: a case series and literature review. eNeurologicalSci 2021;24:100350.

11. Shikata J, Yamamuro T, Iida H, Sugimoto M. Surgical treatment for symptomatic spinal adhesive arachnoiditis. Spine (Phila Pa 1976) 1989;14:870-5.

12. Tachibana T, Moriyama T, Maruo K, Inoue S, Arizumi F, Yoshiya S. Subarachnoid-subarachnoid bypass for spinal adhesive arachnoiditis. J Neurosurg Spine 2014;21:817-20.

13. Ginanneschi F, Palma L, Rossi A. Arachnoid cyst and arachnoiditis following idiopathic spinal subarachnoid haemorrhage. Br J Neurosurg 2008;22:578-9.

14. Velz J, Fierstra J, Regli L, Germans MR. Spontaneous spinal subarachnoid hemorrhage with development of an arachnoid cyst-a case report and review of the literature. World Neurosurg 2018; 119:374-80. 